\title{
Counseling Attitudes and Stigma among Polynesian Americans
}

Timothy B. Smith

Brigham Young University, tbs@byu.edu

G. E. Kawika Allen

Brigham Young University, gekawika_allen@byu.edu

Ofa Hafoka

Brigham Young University

Follow this and additional works at: https://scholarsarchive.byu.edu/facpub

Part of the Counseling Psychology Commons

\section{Original Publication Citation}

Allen, G. E., Kim, B., Smith, T. B., \& Hafoka, O. (2016). Counseling attitudes and stigma among Polynesian Americans. The Counseling Psychologist, 44, 6-27.

\section{BYU ScholarsArchive Citation}

Smith, Timothy B.; Allen, G. E. Kawika; and Hafoka, Ofa, "Counseling Attitudes and Stigma among Polynesian Americans" (2016). Faculty Publications. 1992.

https://scholarsarchive.byu.edu/facpub/1992

This Peer-Reviewed Article is brought to you for free and open access by BYU ScholarsArchive. It has been accepted for inclusion in Faculty Publications by an authorized administrator of BYU ScholarsArchive. For more information, please contact ellen_amatangelo@byu.edu. 
Allen, G. E., Kim, B., Smith, T. B., \& Hafoka, O. (2016). Counseling attitudes and stigma among Polynesian Americans. The Counseling Psychologist, 44, 6-27.

Counseling Attitudes and Stigma among Polynesian Americans 


\section{Abstract}

There is a paucity of research on the mental health of Pacific Islanders living in the U.S., including those of Polynesian descent. This study examined coping strategies, attitudes towards seeking mental health counseling, public and self-stigma towards seeking professional help, and psychological adjustment among 638 Polynesian Americans. On average, participants held neither favorable nor unfavorable attitudes towards seeking professional psychological help, but Polynesian American men endorsed moderate self-stigma about mental illness and Polynesian American women endorsed high levels of public stigma about mental illness. Women showed relatively more favorable attitudes than men about seeking help from professional mental health providers. Participants reported benefitting from culturally congruent practices for coping with distress (accepting, reframing, striving, family support, and religiosity/spirituality) much more than seeking assistance from private emotional outlets (e.g., therapy) or avoidance and detachment. Findings indicate that mental health professionals will need to build effective crosscultural bridges and culturally adapt services to address concerns among Polynesian Americans.

Keywords: counseling attitudes, mental health stigma, Polynesian Americans 
Counseling Attitudes and Stigma among Polynesian Americans

\section{Introduction}

For a variety of reasons, individuals who experience emotional distress or mental illness may not seek professional mental health services to alleviate those conditions. A large body of empirical research has documented public and self-stigma towards seeking professional help and other factors such as gender roles that may promote or discourage individuals from seeking psychotherapy and related services (Cepeda-Benito \& Short, 1998; Golberstein, Eisenberg \& Gollust, 2008; Link, Cullen, Frank \& Wozniak, 1987; Mackenzie, Gekoski \& Knox, 2006; Watson, Corrigan, Larson \& Sells, 2007; Vogel, Wade \& Hackler, 2007). Underutilization of professional mental health services due to stigma and other barriers is particularly problematic among people of color in North America (Sue, Cheng, Saad, \& Chu, 2012; Alvidrez, 1999; Diala et al, 2000; Duncan, 2003; Nickerson, Helms \& Terrell, 1994). The U.S. Surgeon General (1999) and a follow-up government report (2001) called attention to racial inequities in mental health service utilization, with a recent meta-analysis of data from over 300,000 individuals (Smith \& Trimble, 2015) confirming racial discrepancies in mental health service utilization.

Racial and ethnic minorities underutilize professional mental health services for several reasons, including cultural mistrust and cultural attitudes relevant to seeking help from others (Diala, Muntaner, Walrath, Nickerson, LaVeist \& Leaf, 2000; Duncan, 2003; López, Barrio, Kopelowicz \& Vega, 2012; Nickerson, Helms \& Terrell, 1994; Snowden, 2012). For instance, both Latina and African American women endorse the cultural value of discussing problems within the family and not disclosing them to others outside the home (Alvidrez, 1999). Similarly, Asian Americans' adherence to traditional cultural values predicts unfavorable attitudes toward seeking professional psychological help (Kim \& Omizo, 2003; Sue, Cheng, Saad, \& Chu, 2012). 
Thus mental health professionals’ understanding of and effective responses to cultural values are essential to bridging the current divide in service utilization (Smith \& Trimble, 2015).

A factor associated with the underutilization of mental health services by people of color that has repeatedly received attention in the research literature is cultural stigma about those services (e.g., Garrett \& Portman, 2011; King, Trimble, Morse, \& Thomas, 2014). These cultural beliefs include public stigma and self-stigma. When such stigma about professional mental health treatments combine with experiences of racism, such as racial discrimination experienced in a mental health clinic by a person of color with a mental disorder, the result has been called double stigma (Gary, 2005). People who experience racism and other forms of prejudice appear more likely to develop and maintain coping strategies for handling distress that do not depend on disclosure to strangers, including mental health professionals, with several research studies finding higher rates of stigma among people of color compared with White/European Americans (e.g., Eisenberg, Downs, Golberstein \& Zivin, 2009; Nadeem et al., 2007).

The vast majority of the research on cultural stigma of mental health services has involved African Americans, Asian Americans, and Hispanic/Latino(a) Americans (Allen \& Heppner, 2011), but research specific to other ethnic groups has been extremely limited. Other ethnic groups, including Pacific Islander Americans, have culturally-based coping strategies and beliefs about mental health and mental health services that have not been adequately investigated in the research literature. Thus we presently know very little about Pacific Islander Americans' perceptions of professional mental health services and the cultural factors that underlie those beliefs. In the past, when researchers conducted psychological research with Pacific Islander populations, they tended to lump these groups with Asian American populations, a highly problematic practice that originated with the U.S. Census Bureau and continued until the 2000 
Census. For decades, the illogical combining of these two broad populations (with completely distinct geographic, linguistic, socioeconomic, and cultural origins) resulted in a dearth of information specific to the psychological characteristics of Pacific Islanders, including their helpseeking attitudes, stigma about mental illness, and preferred coping methods.

Furthermore, just as individuals with ancestry originating from dozens of distinct ethnic groups in Asia (e.g., Bhutanese, Han, Hmong, Mongolian, Punjabis) cannot reasonably be understood by researchers who combine them into a single group of Asian Americans (Leong et al., 2007), individuals having ancestry from one or more of hundreds of islands spread across thousands of miles in the Pacific Ocean cannot be accurately understood by the use of the broad categorization, Pacific Islander. That umbrella term includes three completely distinct groups of people from Micronesia (e.g., Guamanians), Polynesia (e.g., Samoans), and Melanesia (e.g., Fijians). Yet these differences are rarely considered. Among many examples that could be given, a recent landmark report on racial and ethnic differences in mental health service use (SAMHSA, 2015) reported the broad category of "Native Hawaiian or Other Pacific Islander.” Although clearly preferable to the completely worthless combination of Pacific Islanders with people of Asian origin, continued over-simplification in research perpetuates the unfortunately normative ethnic gloss (Trimble \& Bhadra, 2013) and fails to provide specific information about why individuals from distinct ethnic backgrounds underutilize mental health services in North America.

Polynesian Americans are among the many groups that are significantly underrepresented in the research literature and underserved in the mental healthcare system. This uniquely diverse subgroup of Pacific Islanders has ancestral origins in the Polynesian triangle of islands (Barcham, Scheyvens \& Overton, 2009). The triangle, which stretches from New Zealand to 
Easter Island and then north to Hawai'i, consists of many nations and island groups, including Hawai’i, Tonga, American Samoa, Western Samoa, Tahiti, Cook Islands, French Polynesia, Niue, Easter Island, and Marquesas Islands. The word Polynesian comes from the Greek roots polus (many) and nesos (islands), and is the preferred form of identification by those who come from these island groups (Capstick, Norris, Sopoaga \& Tobata, 2009; Firth, 2013). Approximately 1.2 million Polynesian Americans reside in the U.S., $0.4 \%$ of the U.S. population (U.S. Census, 2010). Polynesian Americans were one of the fastest growing ethnic groups in the U.S. between 2000 and 2010 (U.S. Census, 2010).

Several cultural values that are common to Polynesians and Polynesians Americans have direct relevance for their methods for coping with mental illness and their attitudes about seeking professional treatment. Polynesian Americans typically embrace the value of collectivism as a strong component of their cultural identity. Very close interpersonal relationships are maintained in a cultural context of social interconnectedness (Singelis, 1994) that emphasizes collective goals over individual ambitions. Polynesian populations hold values based on religion/spirituality, pluralism, and communalism that inform their health-seeking behaviors (Capstick, Norris, Sopoaga \& Tobata, 2009; Firth, 2013). Socialization and cultural interpretations of illness as personal weakness play a major role in help-seeking behaviors. Specific cultural practices may also be involved in the healing process, such as in the Hawaiian healing tradition of Ho'oponopono, which encompasses enabling the person to "set or make things right” or to correct or restore and maintain healthy relationships with family members and others through prayer, discussion, confession, repentance, mutual restitution, and forgiveness (Puku'i, 1972). Cultural values and associated religious/spiritual beliefs shape the kinds of psychological coping methods used by Polynesian Americans, and they are highly likely to 
utilize those culturally appropriate methods to deal with psychological adjustment, depression, anxiety, and stress (Allen \& Smith, 2015).

The entrenched collectivism of people of Polynesian origin arises from tight family units that are the foundational and central focus of activities and worldviews (Handy \& Puku'i, 1950; Puku'i, 1989). Polynesian cultures thus designate the family as the main source of help and coping with emotional struggles (Allen \& Heppner, 2011). In many Polynesian families, seeking help for emotional struggles outside of the family may bring shame and dissatisfaction within the family, along with a sense of personal weakness. Maintaining deep respect for the family name is paramount, with personal weakness that can incur dishonor causing strain in interpersonal relationships. Such an intense focus on family and collective interests can conflict with the bias of individualism that characterizes most contemporary mental health practices (Kirmayer, 2007). Hence there is a clear need for scholars to help bridge the cultural gap between Polynesian worldviews and professional mental health services.

Despite the general lack of research specific to Polynesian Americans, some investigations of psychological variables with that population have been conducted (e.g., McCubbin, Ishikawa, \& McCubbin, 2008; McCubbin \& Marsella, 2009). For instance, strength of individuals’ Polynesian ethnic identity has been linked to higher self-esteem and lower endorsement of depression and anxiety, with collectivistic coping strategies such as family support and religiosity/spirituality buffering the effects of trauma and stressful events (Allen, Garriott, Reyes \& Hsieh, 2013; Allen \& Heppner, 2011; Allen \& Smith, 2015). Much more research is needed to understand how collectivistic coping strategies and stigma about mental illness relate with Polynesian Americans’ willingness to seek professional services. Such research could aid the psychology profession to culturally adapt practices to increase rates of 
treatment utilization, retention, and outcomes (Smith \& Trimble, 2015).

We therefore sought to investigate Polynesians’ help-seeking attitudes and psychological adjustment as a function of their reliance on collectivistic coping strategies and their public and private stigma about emotional distress and mental illness. Similar research with other ethnic minority populations (e.g., Chang, 2008) has found that support from family members can be more protective against mental illness than professional and peer support, particularly when participants experience mental health stigma (Lindsey, Joe, \& Nebbitt, 2010). Previous research with other populations has also evaluated mediation pathways, with personal negative beliefs about emotional distress and mental illness (self-stigma) shown to mediate the association between negative cultural/social beliefs about mental illness (public stigma) and attitudes about receiving professional treatment (Vogel, Wade, \& Hackler, 2007). We sought to evaluate the degree to which those findings characterize Polynesian Americans.

The rationale for this study was based on the Social Behavior Model (Anderson, 1995), which was originally developed by Anderson and Newman (1973) to explain why people utilize or underutilize health services. They described several predisposing factors that facilitate and impede service utilization, including cultural and geographic variables, as well as personal attributes such as gender and social networks. The theory places a strong emphasis on the influence of cultural norms and values, including how those norms differ by gender, so we planned to evaluate level of collectivistic coping and stigma by participant gender. Given the theoretical model that guided this study, as well as the results of prior research findings, we investigated the following research hypotheses:

Hypothesis 1: Due to cultural values that emphasize emotional strength and reliance on family support, Polynesian Americans will exhibit (1) negative attitudes towards seeking 
professional mental health services, (2) negative self-stigma about seeking those services, and (3) negative public-stigma about mental illness in general. Given the tenets of the Social Behavior Model and traditional Polynesian gender roles, men will exhibit greater negative attitudes about seeking help and higher levels of self-stigma and public-stigma concerning mental illness than women.

Hypothesis 2: Due to collectivistic cultural values, family support and religiosity/spirituality will be the most common coping strategies reported by Polynesian Americans, with women and men reporting equivalent use of those coping strategies.

Hypothesis 3: Due to cultural values suggesting that emotional difficulties indicate personal weakness, Polynesian Americans’ reports of depression, anxiety, and stress will be positively correlated with self-stigma but will be negatively correlated with public stigma and attitudes about help-seeking. The use of culturally appropriate collectivistic coping strategies (acceptance, reframing, striving, family support, and religiosity/spirituality) will be negatively correlated with depression, anxiety, and stress, but the coping strategies of avoidance, detachment, and use of private emotional outlets will be positively correlated with depression, anxiety, and stress.

Hypothesis 4: Due to Polynesian Americans’ perceptions of personal psychological struggles as a sign of weakness, self-stigma regarding mental illness will mediate the relationship between public-stigma and attitudes about seeking professional services.

\section{Method}

\section{Participants}

The sample for this study included 638 Polynesian American individuals (386, 60\% women; 252, 40\% men) residing primarily in California, Nevada, Utah, Washington, Arizona, 
Missouri, and Hawai'i. The sample included the following subgroups of Polynesian Americans: Tongan (221; 35\%), Samoan (143; 22\%), Native Hawaiian (64; 10\%), Fijian (39; 6\%), Tahitian (24; 4\%), Maori (15; 2\%), and multi-ethnic Polynesian heritage (132; 21\%). Ninety-eight percent of these Polynesian Americans reported a Christian religion background (e.g., Catholic, Protestant, Baptist, Latter-day Saint). Their ages ranged from 18 to 76 years, with a mean of 30 years. Regarding highest education level, 14\% earned a high school diploma level, $40 \%$ attended some college, $25 \%$ received a 4 -year degree, $7 \%$ received a master’s degree, and $1.3 \%$ received a doctorate-level degree (e.g., $\mathrm{PhD}, \mathrm{MD}, \mathrm{JD})$.

\section{Instruments}

\section{Attitudes Toward Seeking Professional Psychological Help Scale (ATSPPH; Fischer}

\& Farina, 1995). ATSPPH is a 10-item scale that was revised from the original 29-item measure (Fischer \& Turner, 1970). It assesses peoples’ attitudes about seeking professional psychological help (e.g., "If I believed I was having a mental breakdown, my first inclination would be to get professional attention.”). Items are scored from 1 (disagree) to 4 (agree); higher scores indicate more positive attitudes. The revised and original scales are significantly correlated at .87, which suggests strong construct validity (Fischer \& Farina, 1995). Strong internal consistency was found with Cronbach's alpha at .84 and 1-month test-retest at .80 reliabilities have been reported for college student samples. In another study using this measure, the internal consistency was .80 (Vogel, Wade, \& Hackler, 2007). For the present study, the Cronbach’s alpha was .70.

Perceived Devaluation-Discrimination Scale (PDDS). The PDDS (Link et al., 1989) is a 12-item scale that measures perceived public stigma (e.g., "Most people believe that entering a mental hospital is a sign of personal failure” and "Most people would not hire a former mental patient to take care of their children, even if he or she had been well for some time”). Participants 
endorse scores from 1 (strongly disagree) to 6 (strongly agree) indicating belief statements about how most people view current or former psychiatric patients. A higher score indicates greater perceived public stigma. Internal consistency ranged from .76 to .88 among clinical and community samples (Link et al., 1989, 2001; Vogel, Wade, \& Hackler, 2007). For the present study, the Cronbach’s alpha was .75.

Self-Stigma of Seeking Help Scale (SSOSH). The SSOSH (Vogel et al., 2006) is a 10item measure of self-stigma that consists of items such as "I would feel inadequate if I went to a therapist for psychological help," “Seeking psychological help would make me feel less intelligent,” "I would feel worse about myself if I could not solve my own problems,” "It would make me feel inferior to ask a therapist for help.” It is scored on a scale ranging from 1 (strongly disagree) to 5 (strongly agree) with higher scores indicating greater self-stigma. Studies have shown internal consistency ranging from .86 to .90, and a 2-week test-retest reliability has been reported to be .72 (Vogel et al., 2006; Vogel, Wade, \& Hackler, 2007). For the present study, the Cronbach’s alpha was .82.

Collectivistic Coping Styles Scale (CCS). The CCS (Heppner et al., 2006) is a 30-item measure of coping strategies used in collectivistic societies that was developed and validated in three studies with Taiwanese college students. In those studies, five factors were identified through exploratory and confirmatory factor analyses: (a) Acceptance, Reframing, and Striving (ARS); (b) Family Support (FS); (c) Religion-Spirituality (RS); (d) Avoidance and Detachment (AD); and (e) Private Emotional Outlets (PEO). The CCS is rated on scale from $0=$ Never used this strategy/Not applicable, $1=$ Used but of no help at all, $2=$ A little help, $3=$ A moderate amount of help, 4 = A great deal of help, to $5=$ A tremendous amount of help. A higher score indicates greater helpfulness for the particular coping strategy. The Cronbach alpha coefficient 
was .87 when scale was initially validated. For each of the scores for the present study, the Cronbach’s alpha was .83 (ARS), .86 (FS), .87 (RS), .85 (AD), .76 (PEO), and .91 for the entire scale.

Depression Anxiety Stress Scale-21 (DASS-21). The DASS-21 (Lovibond \& Lovibond, 1995) contains the following three subscales with each subscale containing seven items: Depression, Anxiety, and Stress. Sample items are: Depression (e.g., "I felt downhearted and blue”); Anxiety (e.g., "I felt I was close to panic”); and Stress (e.g., "I found it hard to wind down”). The DASS-21 assesses the emotional levels of depression, anxiety, and stress over the past week and is rated on a 4-point scale ranging from 0 (did not apply to me at all) to 3 (applied to me very much, or most of the time). Cronbach's alpha for the DASS-21 ranged from .84 to .91 across subscales, respectively (Lovibond \& Lovibond, 1995). For each of the subscale scores for this study, the Cronbach's alpha was .88 (ANX), .90 (DEP), .87 (STR). For the overall scale, the Cronbach’s alpha was .95.

\section{Procedure}

After securing the IRB approval from the host institution, participants were recruited with announcements distributed nationwide via social media including Facebook, and through emails inviting various influential Polynesian individuals including college students. Participants completed the survey online through Qualtrics, receiving a \$10 gift card as an incentive and compensation for their time. We believed that inviting Polynesian American individuals through personal and social media would be an effective method of collecting data. There were no exclusion criteria for participation in the study. Two doctoral students who assisted in the data collection of this study posted the Qualtrics link on their Facebook profile, which contains roughly 80\% Polynesian American individuals. The response rate was tracked through Facebook 
by asking participants to message back indicating if they had or hadn’t taken the study. That number of those who indicated through Facebook that they had participated in the study corresponded with the Qualtrics number of participants completing the study, resulting in a response rate of $84 \%$.

\section{Results}

\section{Attitudes towards Professional Psychological Services and Stigma about Mental Illness}

The first research hypothesis concerned Polynesian Americans' attitudes towards and stigma about seeking professional psychological services. We evaluated both self-stigma and public stigma towards individuals with mental illnesses, as well as the differences between men and women (Hypothesis 1).

Regarding attitudes towards seeking professional mental health services, the Polynesian American participants had a mean score of $2.6(S D=.27)$ on the 1 to 4 rating of the ATSPPH, which indicated that they had neither favorable nor unfavorable attitudes about seeking psychological help from a professional. According to the distribution of responses, only $6.3 \%$ of Polynesian Americans in the sample indicated highly favorable attitudes (average score of 3 or greater on the 4-point scale) towards seeking professional mental health services, and only $2.5 \%$ reported unfavorable attitudes (average score less than 2 on the 4-point scale). The vast majority of the sample indicated ambivalent attitudes.

A MANOVA examining the differences between Polynesian American men and women reached statistical significance (Wilks' Lambda $\left.=.97, F_{(\mathrm{df}=3)}=7.45, p<.001\right)$. Although their scores were still in the range of ambivalence, Polynesian women endorsed more favorable attitudes towards seeking help from mental health professionals than men, $F(1,637)=9.54, p=$ .002 (see Table 1). 
Regarding self-stigma about seeking professional counseling services, Polynesian Americans as a whole exhibited a mean of $2.7(S D=.64)$ on the 1 to 5 rating of the SSOSH, suggesting that they neither scored high or low on self-stigma towards seeking psychological help. Across all participants, high levels of self-stigma were reported in $7.4 \%$ of the sample, and $16.3 \%$ indicated low levels of self-stigma, with most participants reporting mild to moderate levels of stigma. When comparing differences between men and women, males reported greater negative self-stigma than Polynesian females, $F(1,637)=7.52, p=.006$ (see Table 1 ). These results partially supported our first hypothesis that men would show greater self-stigma about mental illness and that women would be more favorable to seeking professional help.

For public-stigma among Polynesian Americans, the data yielded a mean of $4.0(S D=$ .64) on the 1 to 6 rating of the PDDS, showing that this sample of Polynesian Americans reported perceptions of negative social stigma about mental illness. That is, the participants believed that other people in general devalue people experiencing mental illness, with $34 \%$ of the sample showing high levels of public-stigma and only 3.9\% exhibiting low levels of public stigma. An analysis of gender differences showed that Polynesian women scored significantly higher on public stigma than men, reflecting unfavorable social perceptions of persons with psychological difficulties, $F(1,637)=9.26, p=.006$ (see Table 1 ). We had not predicted that women would endorse more unfavorable public norms about other peoples’ psychological difficulties.

\section{Collectivistic Coping Strategies}

We next examined the common collectivistic coping strategies used by the Polynesian American participants. The average ratings for the collectivistic coping strategies on the 0 to 6 scale of the CCS were as follows: Religion/Spirituality $(M=5.1, S D=.98$, with $87 \%$ of 
participants endorsing this strategy as being helpful); Family Support $(M=4.5, S D=1.1 ; 73 \%$

endorsed as helpful); Acceptance, Reframing, and Striving $(M=4.4, S D=.81 ; 68 \%$ endorsed as helpful); Avoidance and Detachment ( $M=3.4, S D=1.2 ; 26 \%$ endorsed as helpful); and Private Emotional Outlets $(M=2.7, S D=1.3 ; 17 \%$ endorsed as helpful). Thus the sample strongly endorsed religion/spirituality and family support systems, but did not endorse either avoidance/detachment or private emotional outlets as helpful coping methods.

A MANOVA conducted to examine differences in collectivistic coping strategies between Polynesian American men and women (Hypothesis 2) reached statistical significance (Wilks’ Lambda $\left.=.96, F_{(\mathrm{df}=5)}=4.6, p<.001\right)$. Post hoc univariate analyses indicated differences on two of the five collectivistic coping strategies: Women endorsed higher scores on religion/spirituality, $F(1,637)=6.40, p=.012$, and men reported higher endorsement of avoidance and detachment as helpful coping strategies, $F(1,637)=11.33, p=.001$ (see Table 1 ). Although we predicted that religion/spirituality would be reported as the most helpful coping strategy used by Polynesian Americans in general, we had not predicted the finding that women would report greater assistance from religion/spirituality than men. Neither men nor women found avoidance and detachment from psychological struggles to be particularly helpful, but the men were less averse to those coping strategies than women.

\section{Relationships among Stigma, Coping, and Psychological Adjustment}

Zero-order bivariate correlations (see Table 2) showed various statistically significant links between stigma, coping, and psychological indicators (Hypothesis 3). Self-stigma was positively correlated with depression, anxiety, stress, while public stigma was negatively associated with depression and anxiety. Self-stigma was also negatively correlated with attitudes about seeking mental health services. Regarding coping strategies, public stigma was negatively 
associated with private emotional outlets, while self-stigma was positively linked to avoidance/detachment, and public stigma was negatively correlated with family support. Similarly, attitudes towards seeking professional services were negatively correlated with anxiety, depression, and stress. The coping strategies of avoidance/detachment and use of private emotional outlets were positively correlated with anxiety, depression, and stress.

As we predicted in Hypothesis 3, Polynesian Americans’ reports of depression, anxiety, and stress were positively correlated with self-stigma and depression and anxiety were negatively correlated with public stigma. An additional accurate prediction was that attitudes about seeking professional services would be negatively correlated with depression, anxiety, and stress.

Although we had hypothesized that culturally appropriate collectivistic coping strategies (acceptance, reframing, striving, family support, and religiosity/spirituality) would negatively correlate with depression, anxiety, and stress, that hypothesis was not supported. However, the results confirmed the hypothesis that coping strategies of avoidance, detachment, and use of private emotional outlets would be positively correlated with depression, anxiety, and stress.

\section{Evaluation of Self-Stigma as a Mediator of Help-Seeking}

In previous research (Vogel et al., 2007) self-stigma has been found to mediate the association between public-stigma and willingness to seek counseling. We sought to evaluate a similar model examining a possible mediated relationship with those variables (Figure 1;

Hypothesis 4). Based on recent mediation analysis research (Allen \& Wang, 2014; Allen, Wang \& Stokes, 2015) and recommendations for running mediation analyses (Hayes, 2013), we used the SPSS macro PROCESS to conduct the mediation analysis. PROCESS offers bootstrap estimates to calculate bias-corrected confidence intervals for the mediation effects. This bootstrapping method, which has advantages over the causal steps approach (Baron \& Kenny, 
1986) and the Sobel (1982) test, has been recommended as the method of choice for testing indirect effects (Hayes, 2009). The procedure, which involves resampling multiple times and estimating the sampling distribution from all the resamples, enhances estimation accuracy of the indirect effects (Preacher \& Hayes, 2008; Allen, Wang \& Stokes, 2015). In the present study, we estimated the mean of 95\% confidence intervals (CI) of indirect effects derived from 10,000 bootstrap samples. If the upper and lower bounds of the CI do not include zero, then the presence of an indirect effect can be concluded with $95 \%$ confidence. Contrary to our hypothesis, there was a very weak association between public-stigma and attitudes about seeking professional services (Table 4), and self-stigma did not mediate that association (95\% CI [-.01, .009]; indirect effect $=-.03$, S.E. $=.01, p<.707$; total effect $=-.009$, S.E. $=.01, t=-.86, p=.387$; direct effect $=$ -.20$, S.E. $=.01, t=-13.34, p<.001)$.

\section{Discussion}

This study sought to examine Polynesian Americans' attitudes toward seeking professional psychological help and their experiences with self-stigma, public stigma, coping strategies, and psychological distress. Given the paucity of research on these constructs with Polynesian Americans, we first sought to describe how the participants rated the constructs and secondly to determine how their experiences represented by these constructs may be interrelated.

Participants’ attitudes towards seeking professional psychological help were neutral, neither favorable nor unfavorable. Although still holding attitudes in the neutral range, Polynesian American women endorsed more favorable attitudes towards seeking help from mental health professionals than did their male counterparts. Overall ratings on self-stigma were also neutral, and women experienced less self-stigma than did men. The neutral attitudes about seeking professional psychological help did not support Hypothesis 1, which predicted 
unfavorable attitudes among Polynesian Americans due to their cultural and ethnic values emphasizing family support as a primary source of emotional help. Some Polynesian Americans may be open to seeking additional support and guidance outside of their family, and ways to facilitate the utilization of professional mental health services should be explored in future research.

Polynesian American participants tended to believe that society in general negatively perceives individuals with mental illness, with women reporting greater public-stigma than men. These results indicated an important difference between Polynesian American men and women. Polynesian American men tended to be less comfortable than women in disclosing their personal struggles with professional mental health therapists, but women perceived greater public-stigma regarding mental illness. Although Polynesian American men may not see themselves as having psychological problems and may be reluctant to seek professional help, they do not necessarily view others entirely negatively when they experience mental illness. On the other hand, Polynesian American women are somewhat more open to seeking psychological help and to acknowledging their own emotional difficulties, but they tend to devalue or view others critically when they experience mental illness. As suggested by the Social Behavior Model, gender interacts with culture in shaping views toward mental illness and help-seeking.

With respect to preferences for emotional coping strategies, participants most strongly endorsed the use of religion and spirituality, followed by seeking help from family members. This finding supported our second research hypothesis. Although both women and men strongly endorsed religion and spirituality as coping mechanisms, Polynesian American women in particular tended to use religion and spirituality at extremely high rates. The fact that Polynesian Americans most often find religion and spirituality to be helpful is consistent with cultural 
values, with religion playing an important role in that society and with over $96 \%$ of Polynesians in the world professing Christianity (Association of Religion Data Archives, 2015). Therapists will likely benefit from discussing issues pertinent to client experiences with religion and spirituality (e.g., Richards \& Bergin, 2014).

In addition to seeking religious and spiritual sources of healing, participants relied on family support as well as the strategy of accepting the problem, reframing it, and striving to overcome it. Given that many Polynesian American families operate within a collectivistic system (Allen \& Heppner, 2011; Allen \& Smith, 2015), it makes sense that they would utilize strong interpersonal relationships among family members to resolve problems. In contrast, the participants were least likely to endorse the use of private emotional outlets, which would include counseling and other individualistic methods for handling challenges. Thus any attempt by counselors to provide support for Polynesian American individuals should recognize how that individual views counseling relative to other, more collectivistic options - and seek ways to make psychotherapy more inclusive of a client's social circles than would otherwise be the case in treatment as usual.

The results of correlational analyses among stigma, coping, and psychological adjustment show that increased self-stigma was associated with greater psychological distress and less positive attitudes about seeking psychological help, as partly predicted (Hypothesis 3). These results are consistent with previous findings from other populations (Vogel et al., 2007) and suggest that Polynesian American individuals who have high levels of self-stigma are more atrisk for mental health problems, and when they do experience these symptoms, they are less likely to seek help. 
Based on the findings of Vogel and colleagues (2007), we had attempted to examine whether self-stigma mediated the relationship between public stigma and attitude toward seeking professional psychological help (Hypothesis 4). However, public-stigma was unrelated to attitudes towards seeking services. In fact, public stigma did not correlate with either self-stigma or attitudes toward seeking professional psychological help, but it was associated with lower levels of depression and anxiety (Table 2). These findings were unexpected because previous literature with other populations (e.g., Vogel et al.) has shown that increased public stigma is associated with elevated mental health symptoms, self-stigma, and unwillingness to seek help. The absence of a presumably strong association between public perceptions and individuals' behaviors (especially in the context of a collectivistic culture) makes these findings puzzling. Interpretation of the findings would be facilitated by future examinations of how social perceptions among Polynesian Americans relate with individuals' self-perceptions and their willingness to seek assistance from others. For instance, Polynesian Americans who experience elevated symptoms of anxiety and/or depression could possibly feel more socially disconnected if public stigma results in social isolation (e.g., withdrawal or ostracization from the family support typically characterizing their peers). However, in this sample, there was no association between family support and psychological distress, possibly indicating that families did not isolate the individuals experiencing emotional distress (see also Stice, Ragan, \& Randall, 2004).

Given the several unexpected findings, including the higher rate of public-stigma among women, the construct of public-stigma needs to be examined in terms of Polynesian American cultural worldviews. It could be that the notion of public-stigma may be experienced differently among Polynesian Americans relative to other populations, with the complex interplay between selfperception and social perception requiring greater explication. 
In the present sample, participants who endorsed the coping strategies found unhelpful by their peers were more likely to report symptoms of psychological distress. That is, individuals who reported coping with problems through avoidance, detachment, and private emotional outlets reported higher levels of anxiety and depression. It stands to reason that individuals using coping strategies perceived by others to be unhelpful would be less effective in handling challenges. Psychologists can attune to the types of strategies being used by their clients and explore alternatives when those strategies do not facilitate improved functioning.

As with all survey studies, several limitations characterize the present results. First, although the present study's sample size was large, Tongans, Samoans, and Native Hawaiians were over-represented and comprised two-thirds of the sample. Hence, the results may not generalize to Polynesian American individuals who were not well represented in the present sample (e.g., Maori). In future studies, more diverse samples should be sought that better represent all Polynesian American ethnic groups. Second, the study was a cross-sectional correlational study. Hence, no causal statements can be made regarding the findings.

Notwithstanding these limitations, the findings have several important implications. Similar to past findings (e.g., Vogel et al, 2007), self-stigma was significantly correlated with attitudes toward seeking professional psychological help, with self-stigma being particularly stronger for Polynesian American males than females. Service providers who work with Polynesian Americans should be mindful of the important role that self-stigma plays in terms of help-seeking behaviors and attitude, and they should seek to correct inaccurate assumptions about mental health services and to provide positive examples of cultural congruence by explicitly informing treatment with cultural values (Smith \& Trimble, 2015). In particular, the results confirming the importance of family support and religion and spirituality should 
encourage therapists to explicitly address those issues in treatment (e.g., Cervantes \& Parham, 2005; Richards \& Bergin, 2014). Although traditional methods of psychotherapy typically focus on the individual without involving others in that person's social networks, the collectivistic nature of Polynesian family systems and religious congregations make it essential for practitioners to incorporate those networks in their own interpretations of the client's circumstances and in the treatment provided, such as conducting family therapy when indicated and seeking consultation or client support from local religious leaders. Religious and community leaders could also provide key sources of collaboration when reaching out to individuals in need of mental health services who are nevertheless unlikely to seek those services on their own.

In sum, mental health professionals should carefully consider the distinct needs, experiences, and cultural worldviews of Polynesian Americans. To better meet the mental health needs of Polynesian Americans, the current gaps in mental health service utilization and the dearth of available research-based information must now be overcome. Practitioners should understand and work to reduce cultural stigmas associated with emotional struggles. And they should attend to cultural values and build upon Polynesian Americans’ preferred methods for handling psychological difficulties, particularly spirituality/religiosity and family support. Researchers should avoid combining Polynesian Americans with people from completely distinct cultural backgrounds and should report cultural differences when they occur. In short, the goal for the future is a psychology inclusive of Polynesian Americans. 


\section{References}

Abe-Kim, J., Takeuchi, D. T., Hong, S., Zane, N., Sue, S., Spencer, M. S., ... \& Alegría, M. (2007). Use of mental health-related services among immigrant and US-born Asian Americans: results from the National Latino and Asian American Study. American Journal of Public Health, 97(1), 91-98.

Allen, G. E. K., Wang, K., \& Stokes, H. (2015). Examining legalism, scrupulosity, family perfectionism and psychological adjustment among LDS individuals. Mental Health, Religion \& Culture.

Allen, G. E., \& Smith, T. B. (2015). Collectivistic coping strategies for distress among Polynesian Americans. Psychological services, 12(3), 322.

Allen, G. E. K. \& Wang, K. (2014). Examining aspects of religiosity, perfectionism, scrupulosity, and well-being among LDS individuals. Psychology of Religion and Spirituality, 6, 3, 257-264.

Allen, G. E. K., Garriott, P. O., Reyes, C. J. \& Hsieh, C. (2013). Racial identity, phenotype, and self-esteem among biracial Polynesian/White individuals. Family Relations, 62, 8291.

Allen, G. E. K. \& Heppner, P. P. (2011). Religiosity, coping, and psychological well-being among Latter-Day Saint Polynesians in the U.S. Asian American Journal of Psychology, $2,1,13-24$

Alvidrez, J. (1999). Ethnic variations in mental health attitudes and service use among lowincome African American, Latina, and European American young women. Community Mental Health Journal, 35(6), 515-530.

Anderson, R. M. (1995). Revisiting the behavioral model and access to medical care: does it 
matter? Journal of Health and Social Behavior, 36, 1, 1-10

Andersen, R. M. \& Newman, J. F. (1973). Societal and individual determinants of medical care utilization in the United States. Milbank Memorial Fund Quarterly, 51, 95-124

Association of Religion Data Archives (2010). Polynesia. Retrieved May 22, 2015, from http://www.thearda.com/internationalData/regions/profiles/Region_14_2.asp

Barcham, M., Scheyvens, R., \& Overton, J. (2009). New Polynesian Triangle: Rethinking Polynesian migration and development in the Pacific. Asia Pacific Viewpoint, 50(3), 322337.

Baron, R. M., \& Kenny, D. A. (1986). The moderator-mediator variable distinction in social psychological research: Conceptual, strategic, and statistical considerations. Journal of personality and social psychology, 51(6), 1173.

Capstick, S., Norris, P., Sopoaga, F., \& Tobata, W. (2009). Relationships between health and culture in Polynesia-A review. Social Science \& Medicine, 68(7), 1341-1348.

Cepeda-Benito, A., \& Short, P. (1998). Self-concealment, avoidance of psychological services, and perceived likelihood of seeking professional help. Journal of Counseling Psychology, 45(1), 58.

Cervantes, J. M., \& Parham, T. A. (2005). Toward a meaningful spirituality for people of color: lessons for the counseling practitioner. Cultural Diversity and Ethnic Minority Psychology, 11(1), 69.

Chang, H. (2008). Help-seeking for stressful events among Chinese college students in Taiwan: roles of gender, prior history of counseling, and help-seeking attitudes. Journal of College Student Development, 49(1), 41-51. 
Diala, C., Muntaner, C., Walrath, C., Nickerson, K. J., LaVeist, T. A., \& Leaf, P. J. (2000). Racial differences in attitudes toward professional mental health care and in the use of services. American Journal of Orthopsychiatry, 70(4), 455.

Duncan, L. E. (2003). Black male college students’ attitudes toward seeking psychological help. Journal of Black Psychology, 29(1), 68-86.

Eisenberg, D., Downs, M. F., Golberstein, E., \& Zivin, K. (2009). Stigma and help seeking for mental health among college students. Medical Care Research and Review, 66(5), 522-541.

Firth, R. (2013). We the Tikopia: A sociological study of kinship in primitive Polynesia. Routledge.

Fischer, E. H., \& Farina, A. (1995). Attitudes toward seeking professional psychological help: A shortened form and considerations for research. Journal of College Student Development.

Fischer, E. H., \& Turner, J. I. (1970). Orientations to seeking professional help: development and research utility of an attitude scale. Journal of consulting and clinical psychology, 35(1p1), 79.

Garrett, M. T., \& Portman, T. A. A. (2011). Counseling and diversity: Counseling Native Americans. Brooks/Cole.

Gary, F. A. (2005). Stigma: Barrier to mental health care among ethnic minorities. Issues in Mental Health Nursing, 26(10), 979-999.

Golberstein, E., Eisenberg, D., \& Gollust, S. (2008). Perceived stigma and mental health care seeking. Psychiatric Services, 59(4), 392-399.

Handy, E. C., \& Pukui, M. K. (1950). The Polynesian family system in Ka-'u, Hawai'i. The Journal of the Polynesian Society, 232-240. 
Hayes, A. F. (2009). Beyond Baron and Kenny: Statistical mediation analysis in the new millennium. Communication monographs, 76(4), 408-420.

Hayes, A. F. (2013). Introduction to mediation, moderation, and conditional process analysis: A regression-based approach. Guilford Press.

Kim, B. S. K., \& Omizo, M. M. (2003). Asian cultural values, attitudes toward seeking professional psychological help, and willingness to see a counselor. The Counseling Psychologist, 31, 343-361.

King, J., Trimble, J. E., Morse, G. S., Thomas, L. R. (2014). North American Indian and Alaska Native spirituality and psychotherapy (pp. 451-472). In P. S. Richards \& A. E. Bergin (Eds.). Handbook of psychotherapy and religious diversity ( ${ }^{\text {nd }}$ ed.). Washington, D.C.: American Psychological Association.

Kirmayer, L. J. (2007). Psychotherapy and the cultural concept of the person. Transcultural Psychiatry, 44(2), 232-257.

Leong, F. T., Ebreo, A. E., Kinoshita, L. E., Inman, A. G., Yang, L. H. E., \& Fu, M. E. (2007). Handbook of Asian American psychology. Thousand Oaks, CA: Sage.

Lindsey, M. A., Joe, S., \& Nebbitt, V. (2010). Family matters: The role of mental health stigma and social support on depressive symptoms and subsequent help seeking among African American boys. Journal of Black Psychology.

Link, B. G., Cullen, F. T., Frank, J., \& Wozniak, J. F. (1987). The social rejection of former mental patients: understanding why labels matter. American journal of Sociology, 14611500. 
Link, B. G., Cullen, F. T., Struening, E., Shrout, P. E., \& Dohrenwend, B. P. (1989). A modified labeling theory approach to mental disorders: An empirical assessment. American Sociological Review, 400-423.

López, S. R., Barrio, C., Kopelowicz, A., \& Vega, W. A. (2012). From documenting to eliminating disparities in mental health care for Latinos. American Psychologist, 67(7), 511.

Lovibond, P. F., \& Lovibond, S. H. (1995). The structure of negative emotional states: Comparison of the Depression Anxiety Stress Scales (DASS) with the Beck Depression and Anxiety Inventories. Behaviour research and therapy, 33(3), 335-343.

Mackenzie, C. S., Gekoski, W. L., \& Knox, V. J. (2006). Age, gender, and the underutilization of mental health services: the influence of help-seeking attitudes. Aging and Mental Health, 10(6), 574-582.

McCubbin, L. D., Ishikawa, M. E., \& McCubbin, H. I. (2008). The Kanaka Maoli: Native Hawaiians and heir Testimony of Trauma and Resilience. In Ethnocultural perspectives on disaster and trauma (pp. 271-298). Springer New York.

McCubbin, L. D., \& Marsella, A. (2009). Native Hawaiians and psychology: The cultural and historical context of indigenous ways of knowing. Cultural Diversity and Ethnic Minority Psychology, 15(4), 374.

Nickerson, K. J., Helms, J. E., \& Terrell, F. (1994). Cultural mistrust, opinions about mental illness, and Black students' attitudes toward seeking psychological help from White counselors. Journal of Counseling Psychology, 41(3), 378. 
Preacher, K. J., \& Hayes, A. F. (2008). Asymptotic and resampling strategies for assessing and comparing indirect effects in multiple mediator models. Behavior research methods, 40(3), 879-891.

Puku'i, M. K. (1989). The Polynesian family system in Ka-'u, Hawai'i. Tuttle Publishing.

Puku’i, M. K., Haertig, E. W., \& Lee, C. A. (1972). Nana i ke Kumu. Honolulu: Hui Hanai.

Richards, P. S., \& Bergin, A. E. (2014). Religious diversity and psychotherapy: Conclusions, recommendations, and future directions.

Singelis, T. M. (1994). The measurement of independent and interdependent selfconstruals. Personality and Social Psychology Bulletin, 20(5), 580-591.

Smith, T. B., \& Silva, L. (2011). Ethnic identity and personal well-being of people of color: A meta-analysis. Journal of Counseling Psychology, 58(1), 42-60.

Snowden, L. R. (2012). Health and mental health policies' role in better understanding and closing African American-White American disparities in treatment access and quality of care. American Psychologist, 67(7), 524-531.

Stice, E., Ragan, J., \& Randall, P. (2004). Prospective relations between social support and depression: Differential direction of effects for parent and peer support? Journal of Abnormal Psychology, 113(1), 155-159.

Substance Abuse and Mental Health Services Administration, Racial/ Ethnic Differences in Mental Health Service Use among Adults. HHS Publication No. SMA-15-4906. Rockville, MD: Substance Abuse and Mental Health Services Administration, 2015.

Sue, S., Cheng, J. K. Y., Saad, C. S., \& Chu, J. P. (2012). Asian American mental health: A call to action. American Psychologist, 67(7), 532. 
Trimble, J. E., \& Bhadra, M. (2013). Ethnic gloss. In K. Keith (Ed.), Encyclopedia of CrossCultural Psychology, (pp. 500-504). New York: Wiley.

U.S. Department of Health and Human Services (1999). Mental health: A report of the Surgeon General. Rockville, MD: Author.

U.S. Department of Health and Human Services. (2001). Mental health: Culture, race, and ethnicity. Rockville, MD: Author.

Vogel, D. L., Wade, N. G., \& Haake, S. (2006). Measuring the self-stigma associated with seeking psychological help. Journal of Counseling Psychology,53(3), 325.

Vogel, D. L., Wade, N. G., \& Hackler, A. H. (2007). Perceived public stigma and the willingness to seek counseling: The mediating roles of self-stigma and attitudes toward counseling. Journal of Counseling Psychology, 54(1), 40.

Watson, A. C., Corrigan, P., Larson, J. E., \& Sells, M. (2007). Self-stigma in people with mental illness. Schizophrenia bulletin, 33(6), 1312-1318. 
Table 1 Descriptive Statistics and MANOVA for Differences between Gender across Attitudes, Stigma, and Coping for Polynesian Americans $(n=638)$

\begin{tabular}{|c|c|c|c|c|c|}
\hline Measure & $M$ & Std & $F$ & $d f$ & $p$ \\
\hline \multicolumn{6}{|l|}{ Attitudes and Stigma } \\
\hline Attitudes & 2.6 & 27 & 9.54 & 1 & .002 \\
\hline Male & 2.5 & 26 & & & \\
\hline Female & 2.6 & 28 & & & \\
\hline Self-Stigma & 2.7 & .64 & 7.52 & 1 & .006 \\
\hline Male & 2.8 & .59 & & & \\
\hline Female & 2.6 & .66 & & & \\
\hline Public Stigma & 4.0 & .64 & 9.26 & 1 & .002 \\
\hline Male & 3.9 & .56 & & & \\
\hline Female & 4.0 & .67 & & & \\
\hline \multicolumn{6}{|l|}{ Collectivistic Coping Strategies } \\
\hline Acceptance, Reframing, Striving & 4.4 & .81 & 1.28 & 1 & .258 \\
\hline Male & 4.4 & .86 & & & \\
\hline Female & 4.3 & .78 & & & \\
\hline Family Support & 4.5 & 1.1 & .033 & 1 & .856 \\
\hline Male & 4.4 & 1.0 & & & \\
\hline Female & 4.4 & 1.1 & & & \\
\hline Religion/Spirituality & 5.1 & .98 & 6.40 & 1 & .012 \\
\hline Male & 5.0 & 1.1 & & & \\
\hline Female & 5.2 & .91 & & & \\
\hline Avoidance/Detachment & 3.4 & 1.2 & 11.33 & 1 & .001 \\
\hline Male & 3.6 & 1.2 & & & \\
\hline Female & 3.2 & 1.2 & & & \\
\hline Private Emotional Outlets & 2.7 & 1.3 & 2.65 & 1 & .104 \\
\hline Male & 2.7 & 1.3 & & & \\
\hline Female & 2.6 & 1.2 & & & \\
\hline
\end{tabular}

Note: $F$ values refer to post hoc ANOVAs between male and female participants. 
Table 2

Intercorrelations of Study Variables

\begin{tabular}{|c|c|c|c|c|c|c|c|c|c|c|c|}
\hline & 1 & 2 & 3 & 4 & 5 & 6 & 7 & 8 & 9 & 10 & 11 \\
\hline 1.SELSTIG & - & & & & & & & & & & \\
\hline 2.PUBSTIG & .04 & - & & & & & & & & & \\
\hline 3.ATT & $-.47 * * *$ & -.03 & - & & & & & & & & \\
\hline 4. ARS & $-.10^{*}$ & -.04 & .06 & - & & & & & & & \\
\hline 5.FS & -.06 & $-.17^{* * *}$ & $.09 *$ & $.54 * * *$ & - & & & & & & \\
\hline 6.RS & $-.09 *$ & .06 & .04 & $.58 * * *$ & $.53^{* * *}$ & - & & & & & \\
\hline 7.AD & $.18^{* * *}$ & $-.11^{* *}$ & $-.10 * *$ & $.52 * * *$ & $.17 * * *$ & $.13^{* *}$ & - & & & & \\
\hline 8.PEO & -.01 & $-.21 * * *$ & .05 & $.37 * * *$ & $.26^{* * *}$ & $.08 *$ & $.50 * * *$ & - & & & \\
\hline 9.ANX & $.20 * * *$ & $-.14^{* *}$ & $-.14^{* *}$ & $.08^{*}$ & $.09 *$ & -.03 & $.31 * * *$ & $.36 * * *$ & - & & \\
\hline 10.DEP & $.23 * * *$ & $-.15^{* * *}$ & $-.16^{* * *}$ & .05 & .04 & $-.09 *$ & $.28 * * *$ & $.30 * * *$ & $.80 * * *$ & - & \\
\hline 11.STR & $.21^{* * *}$ & -.04 & $-.15^{* * * *}$ & .04 & .02 & -.02 & $.23 * * *$ & $.30 * * *$ & $.80 * * *$ & $.81^{* * *}$ & - \\
\hline
\end{tabular}

Commented [BYU1]: Add explanations for each variable name in a NOTE at the bottom of the table.

Tables must be self-sufficient with all information needed for interpretation. 
Table 3

Regression Results for Mediation: (Public-Stigma $\rightarrow$ Self-Stigma $\rightarrow$ Attitudes)

\begin{tabular}{lccccc}
\hline & & $\mathrm{DV}=$ Attitudes $\left(\mathrm{R}^{2}=.08\right)$ & & & \\
& Predictor & $\boldsymbol{B}$ & $\boldsymbol{S E}$ & $\boldsymbol{t}$ & $\boldsymbol{p}$ \\
\hline Constant & -.03 & .07 & -.864 & .387 \\
Public-Stigma & -.006 & .01 & -.376 & .707 \\
Self-Stigma & .045 & .04 & 1.136 & .256 \\
& Effect & Boot $\boldsymbol{S . E}$ & $\mathbf{9 5 \%}$ CI \\
Indirect effect & -.03 & .01 & {$[.01, .009]$} \\
\hline
\end{tabular}

Note. $N=639$. All $p$ values, two-tailed. $\mathrm{DV}=$ dependent variable; $\mathrm{CI}=$ confidence interval. 


\section{Figure 1}

Mediation model examining the mediating effect of Self-Stigma on the relationship between Public-Stigma and Attitudes about seeking professional services

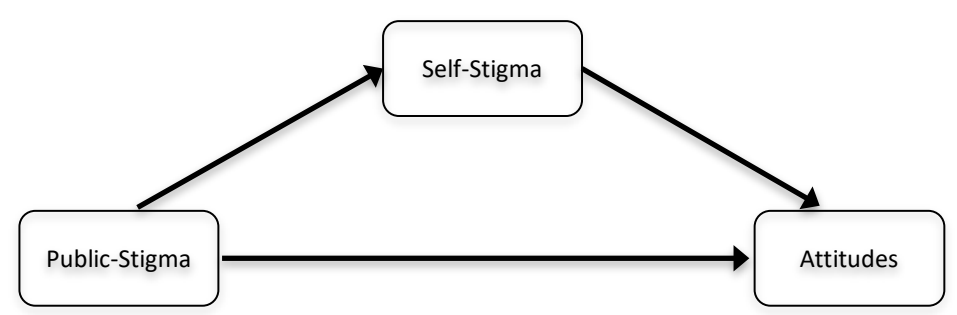

